$<$ Case Report $>$

\title{
A case of hypothyroidism concurrent with myxedema coma in a Cocker spaniel dog
}

\author{
Yun-Hye Kim, Jun-Hwan Kim, Hyun-Seok Lee, Ho-Seong Cho, Jin-Ho Park, Chul Park* \\ College of Veterinary Medicine \& Bio-Safety Research Institute, Chonbuk National University, \\ Jeonju 561-756, Korea
}

(Received 2 May 2012; revised 19 June 2012; accepted 20 June 2012)

\section{Abstract}

A 4-year-old female Cocker spaniel weighing $7.74 \mathrm{~kg}$ was presented with a seven-month history of lethargy and skin problems. On the basis of clinical history, physical examination, laboratory tests, electrocardiogram, radiography, ultrasonography, and thyroid function tests, the dog was diagnosed as hypothyroidism. The dog was administered levothyroxine and presented a good response to the therapy. However, uncontrolled for about 6 months, the dog was re-admitted to hospital with depression and three seizure episodes. The dog was diagnosed as hypothyroidism with concurrent myxedema coma and gallbladder mucocele. With fluid therapy and administration of levothyroxine, the dog was successfully controlled for 3 months after starting treatment. This report describes the clinical features and successful treatment of a Cocker spaniel dog with concurrent hypothyroidism and myxedema coma.

Key words : Hypothyroidism, Hypercholesterolemia, Hyponatremia, Myxedema coma, Levothyroxine

\section{INTRODUCTION}

Hypothyroidism, a common endocrine disorder in dogs, is the result of deficit of the active thyroid hormones, triiodothyronine $\left(\mathrm{T}_{3}\right)$ and thyroxine $\left(\mathrm{T}_{4}\right)$ (Dixon et al, 1999; Mooney, 2011). Hypothyroidism may develop when any part of the hypothalamic-pituitary-thyroid axis is impaired and may be acquired or congenital. Congenital hypothyroidism, which is uncommon in veterinary medicine, can arise as a result of iodine deficiency, dyshormonogenesis, and thyroid dysgenesis (Chastain et al, 1983; Park and Chatterjee, 2005). Acquired hypothyroidism can be primary, secondary, or tertiary. Primary hypothyroidism accounts for most cases of hypothyroidism in dogs and are caused commonly by lymphocytic thyroiditis or idiopathic thyroid atrophy. Other rare forms, secondary and tertiary hypothyroidism, arise from a defect in the pituitary or the hypothalamus and

\footnotetext{
*Corresponding author: Chul Park, Tel. +82-63-270-4873,

Fax.+82-63-270-3780, E-mail. chulpark0409@jbnu.ac.kr
}

the causes include a tumor or surgical extirpation (Graham et al, 2007; Mooney, 2011). Because thyroid hormones are involved in almost any organ system, clinical signs of hypothyroidism may be vague and non-specific, but the most common clinical features are dermatological and metabolic changes (Dixon et al, 1999; Finora and Greco, 2007; Jaggy et al, 1994). Neurologic disorders, cardiovascular abnormalities, myopathies, reproductive abnormalities, and ocular abnormalities also have been reported (Atkinson and Aubert, 2004; Finora and Greco, 2007; Panciera, 2001). Common laboratory findings associated with hypothyroidism are hypercholesterolemia, hypertriglyceridemia, and hyponatremia in serum biochemistry results due to the decrease in normal lipid metabolism, and nonregenerative anemia in complete blood count results (Ferguson, 2007; Graham, 2009; Mooney, 2011). Thyroid function tests are largely helpful for evaluating hypothyroidism but can be influenced by many factors including sick euthyroid syndrome and some drugs. These tests include total $\mathrm{T}_{4}$, free $\mathrm{T}_{4}$, endog- 
enous TSH, antithyroglobulin antibodies, total $\mathrm{T}_{3}$, thyroid-stimulating hormone (TSH) stimulation test, and thyrotropin releasing hormone (TRH) stimulation test (Ferguson, 2007; Graham, 2009).

A rare manifestation of severe hypothyroidism is myxedema coma, which is characterized by altered mentation, hypothermic without shivering, and nonpitting edema. This condition is considered an emergency and may be successfully treated by aggressive supportive therapy and rapid thyroid hormone replacement (Atkinson and Aubert, 2004; Finora and Greco, 2007).

This report describes the clinical, clinicopathological, radiographic, ultrasonographic, and electrocardiographic features and thyroid function test results in a Cocker spaniel dog with hypothyroidism concurrent with myxedema coma, and demonstrates the successful results of immediate and intensive supportive treatment.

\section{CASE REPORT}

A 4-year-old female Cocker spaniel weighing $7.74 \mathrm{~kg}$ was admitted to the Veterinary Medical Teaching Hospital of Chonbuk National University with a 7 month history of lethargy and skin problems. On physical examination, marked mental dullness, bradycardia and dermatologic abnormalities including seborrhea, scaly skin, generalized alopecia, pruritus, and thickened skin were found (Fig. 1). Results of complete blood counts (CBC) revealed normochromic normocytic anemia (HCT $=28.7 \%$; reference range, $37.0 \sim 54.0 \%, \mathrm{MCV}=70 \mu \mathrm{m}^{3}$; reference range, $60 \sim 74 \mu^{3}, \mathrm{MCHC}=34.1 \mathrm{~g} / \mathrm{dl}$; reference range, $32.0 \sim 36.0 \mathrm{~g} / \mathrm{dl}$ ). Results of serum biochemical profiles revealed hypercholesterolemia (Cholesterol $=484 \mathrm{mg} / \mathrm{d}$; reference range, $110 \sim 320 \mathrm{mg} / \mathrm{d}$ ) and mild hyperkalemia $(\mathrm{K}=5.9 \mathrm{mmol} / \mathrm{L}$; reference range, $3.5 \sim 5.8 \mathrm{mmol} / \mathrm{L})$. Skin tests including superficial and deep scraping, taping, dermatophyte test medium culture, and wood lamp examination and radiographic films of abdomen showed no abnormalities. The result of the skin biopsy was consistent with endocrine dermatoses (Fig. 2). On electrocardiogram (ECG), bradycardia with sinus arrest was detected.

Based on these findings, the dog was suspected of
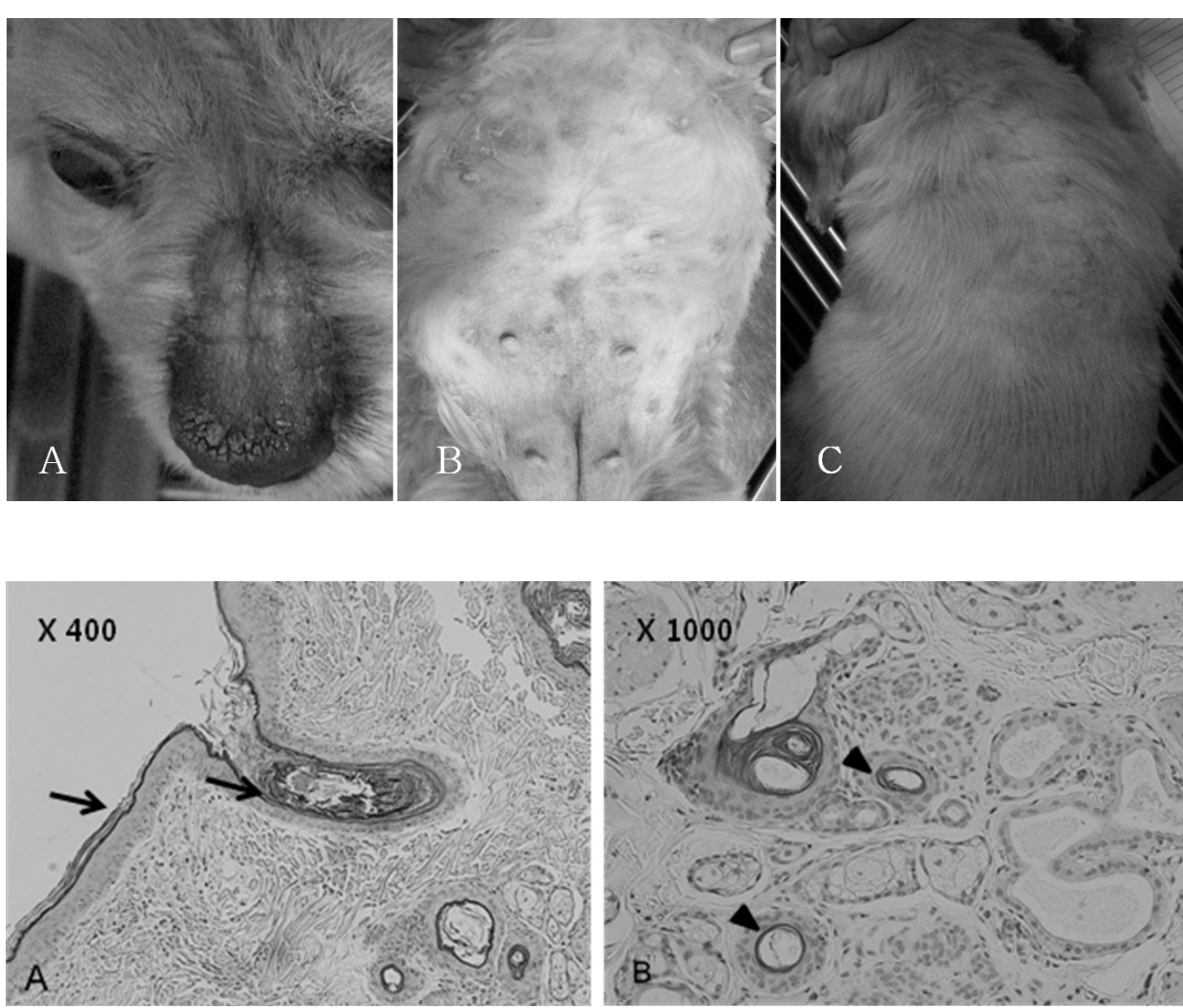

Fig. 1. Dermatologic features of the bridge of the nose (A), the abdomen (B), and the back $(\mathrm{C})$ of the dog. (A) Alopecia and thickened skin were observed. (B, C) Generalized alopecia and dandruffs are present.
Fig. 2. Skin biopsy of the dog. Hyperkeratosis (A; black solid arrows), follicular atrophy (B; Arrow heads), and telogen phase of the hair (B; Arrow heads) were observed. 


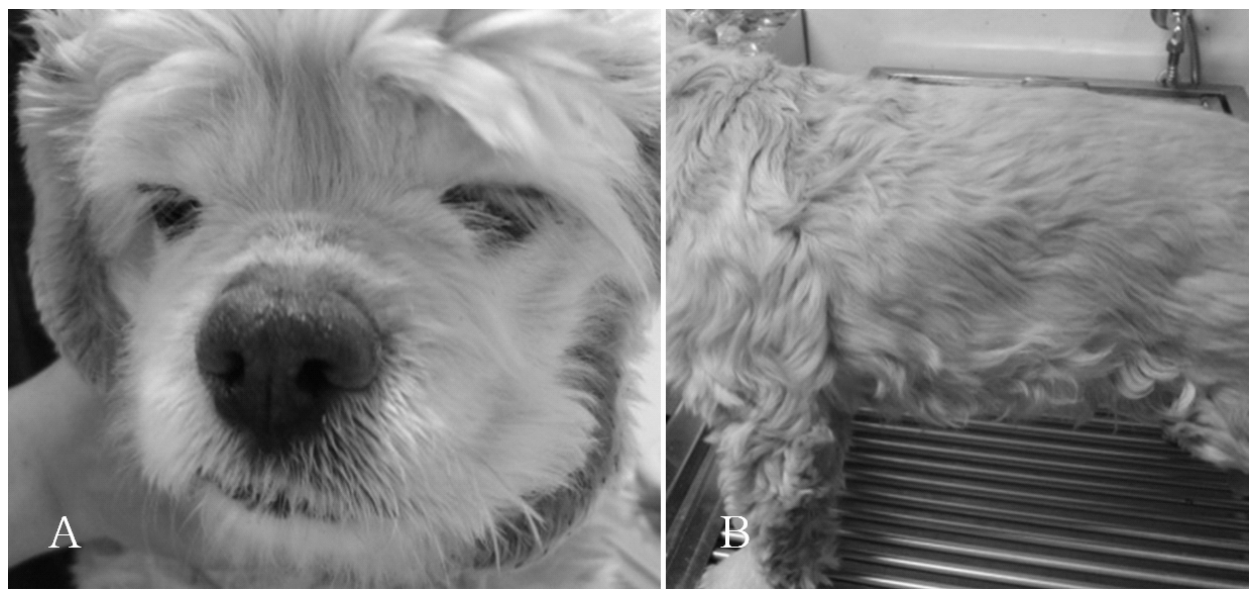

Fig. 3. Dermatologic features of the $\operatorname{dog} 5$ weeks after initiating therapy (A, B). Improvement of dermatologic abnormalities was detected.

having hypothyroidism and thyroid function tests were performed. The concentration of total $\mathrm{T}_{4}$ was $0.7 \mu \mathrm{g} / \mathrm{dl}$ (lower limit range: $0.8 \sim 1.0 \mu \mathrm{g} / \mathrm{dl}$ ) and the concentration of free $\mathrm{T}_{4}$ was $0.02 \mathrm{ng} / \mathrm{dl}$ (lower limit range: $0.5 \sim 0.8$ $\mathrm{ng} / \mathrm{dl})$. TRH stimulation test revealed that the initial concentration of total $\mathrm{T}_{4}$ was $0.8 \mu \mathrm{g} / \mathrm{dl}$, the concentration of TSH 30 minutes after administration of TRH was $0.01 \mathrm{mU} / \mathrm{L}$ (reference range: $0.03 \sim 0.6 \mathrm{mU} / \mathrm{L}$ ), and the concentration of total $\mathrm{T}_{4} 4$ hours after administration of TRH was $0.8 \mu \mathrm{g} / \mathrm{dl}$.

The final diagnosis was made as hypothyroidism and the patient was treated with Levothyroxine (Synthyroid ${ }^{\mathbb{R}}$, Bukwang Pharm Co, Seoul, Korea, $0.02 \mathrm{mg} / \mathrm{kg}$ PO, BID), Seborrhytic shampoo (Sebaclean ${ }^{\circledR}$, Careside Korea, Seoul, Korea), and Benzoyl peroxide (Peroxyderm ${ }^{\circledR}$, Vetoquinol, Poland) for thyroid hormone supplementation and improvement of dermatologic abnormalities, respectively.

The hematocrit value, serum cholesterol level, and concentration of total $\mathrm{T}_{4}$, free $\mathrm{T}_{4}$, and endogenous $\mathrm{TSH}$ were returned to normal range in the second week of therapy. Dermatologic abnormalities improved in 5 weeks (Fig. 3).

Six months after the last visiting without therapy, however, the patient was presented again with a history of anorexia and three times of seizure episodes. On physical examination, mild hypothermia and remarkable depression were found. Neurologic examination revealed delayed proprioception and wheel barrowing in both pel- vic limbs. Decreased facial sensation was also observed. The CBC was within normal limits. Results of serum biochemical profiles noted hypercalcemia (13.2 mg/d; reference range, $7.9 \sim 12.0 \mathrm{mg} / \mathrm{d}$ ), hypercholesterolemia $(>5,200 \mathrm{mg} / \mathrm{d}$; reference range, 110 to $32-\mathrm{mg} / \mathrm{d})$, hyponatremia (142 mmol/L; reference range, $144 \sim 160 \mathrm{mmol} / \mathrm{L})$, and hyperalbunemia $(4.90 \mathrm{mg} / \mathrm{d}$; reference range, $2.3 \sim$ $4.0 \mathrm{mg} / \mathrm{d}$ ). Radiographic findings showed no abnormalities. On abdominal ultrasonography, mucocele is the only significant finding. The concentration of total T4 was lower than $0.4 \mu \mathrm{g} / \mathrm{dl}$ (reference range: $1.0 \sim 4.0 \mu \mathrm{g} / \mathrm{dl}$ ), free $\mathrm{T}_{4}$ was lower than $0.3 \mathrm{ng} / \mathrm{dl}$ (reference range: $0.6 \sim$ $3.7 \mathrm{ng} / \mathrm{dl}$ ), and endogenous TSH was $0.80 \mathrm{ng} / \mathrm{ml}$ (reference range: $0.05 \sim 0.42 \mathrm{ng} / \mathrm{ml}$ ). According to these results, hypothysoidism concurrent with myxedema coma and mucocele was the definitive diagnosis.

During hospitalization, fluid therapy of $0.9 \%$ sodium chloride was continued at $26 \mathrm{~mL} / \mathrm{h}$. Levothyroxine was given. Clemastine $(0.05 \mathrm{mg} / \mathrm{kg}$ PO bid) and Cephalexin (25 mg/kg PO bid) were administrated for antibiotic therapy. Ursodeoxycholic acid (UDCA, $10 \mathrm{mg} / \mathrm{kg}$ PO bid) as choleretics and hepatoprotective drugs was given, as well as S-adenosyl-L-methionine (SAME, $9 \mathrm{mg} / \mathrm{kg}$ PO bid) for antioxidant and hepatoprotective effects. Passive rewarming was used over hours.

Mental state improved in the first week after initiating treatment and the clinical pathologic abnormalities were resolved within 3 weeks. Mucocele was observed unchanged on abdominal ultrasonography. 


\section{DISCUSSION}

Hypothyroidism is a frequently diagnosed endocrine disorder with nonspecific clinical sings. The most common clinical signs are associated with decreased metabolic rate and dermatologic manifestations. A diagnosis of hypothyroidism is made based on clinical signs, physical examination, clinicopathologic findings, and thyroid function tests. Response to treatment was good in most dogs, but those with severe concurrent disease or neurologic abnormalities were less likely to respond with complete resolution of clinical signs (Scott-Moncrieff, 2007).

Of the severe complications, myxedema coma is a rare and severe manifestation of hypothyroidism. The mortality rate for dogs is high, and a positive outcome is highly dependent on early recognition and treatment (Jaggy et al, 1994). Myxedema coma is characterized by changes in mental status, altered thermoregulation, and non-pitting edema of the skin. In this case, hypothermia and marked abnormalities of mental status such as mental depression and seizures were presented. Myxedematous deposition in the brain is responsible for the neurologic changes. Hyponatremia can cause a further development of mentation changes (Fliers and Wiersinga, 2003). Edema formation in the brain may cause alteration in the thermoregulatory center of the hypothalamus, leading to hypothermia without shivering. In addition, in the hypothyroid state, there is decreased ATP use and thus decreased oxygen consumption and decreased heat generation (Atkinson and Aubert, 2004; Blois et al, 2008). Laboratory tests in this case revealed hyponatremia, severe hypercholesterolemia, and a mild, nonregenerative anemia. Decreased cholesterol utilization and a decrease in the number of low density lipoproteins (LDL) receptors of the liver are responsible for hypercholesterolemia in hypothyroidism state. The anemia could be due to a decreased stimulation of erythropoiesis by erythropoietin and thyroid hormones (Finora and Greco, 2007). Thyroid function tests indicated severe hypothyroidism by low total $\mathrm{T}_{4}$, low free $\mathrm{T}_{4}$, and elevated TSH levels.

Myxedema coma is considered as a medical emergency. Once it is diagnosed, immediate treatment is necessary. The goals of treatment include ventilation, passive re- warming, and replacement of endogenous thyroid hormone. In this case, the dog was administered $0.9 \%$ sodium chloride IV at $26 \mathrm{~mL} / \mathrm{h}$ for support of blood pressure and resolution of the hyponatremia. In myxedema coma, intravenous administration of levothyroxine is preferred to oral administration as decreased gastrointestinal motility is seen in hypothyroidismand thus absorption of oral medications may be reduced (Henik and Dixon, 2000). However, it is likely that treatment with levothyroxine IV is uncommon simply because canine myxedema coma is rare. Further studies are needed to determine the dose and risks of levothyroxine IV administration in dogs with myxedema coma (Pullen and Hess, 2006). In this case, oral thyroxine was administered because IV thyroxine was not available.

It has been suggested that gall bladder mucocele (GBM) has relationship with endocrinopathies. A retrospective case-control study showed that the odds of GBM in dogs with hypothyroidism were three times that of dogs without hypothyroidism (Mesich et al, 2009). Management of GBM in this case was attempted by use of antibiotics and choleretics, including clemastine, cephalexin, UDCA, and SAMe.

In many hypothyroidism dogs, successful treatment has been reported. Clinical resolution of metabolic signs can be observed within 2 weeks of starting therapy, dermatological signs resolve up to 3 months, and neurologic deficits are improved rapidly but complete improvement would be achieved in 8-12weeks (Dixon et al, 2002). In myxedema coma dogs and humans, although mortality rates can be high, successful treatment has been reported if immediate and intensive supportive care is made (Atkinson and Aubert, 2004; Finora and Greco, 2007; Yamamoto et al, 1999). In this case report, the affected dog presented improvement of mental status and clinicopathologic abnormalities in a week after initiating therapy, remaining the mucocele unchanged. No evidence of clinical and clinicopathological abnormalities was identified three months after therapy except for the remaining gallbladder on ultrasonography.

In conclusion, the dog in this case was diagnosed with hypothyroidism concurrent with myxedema coma, and immediately fluid therapy and thyroid hormone replacement were initiated. Myxedema coma is extremely 
rare in dogs and the most challenging is recognizing the syndrome (Finora and Greco, 2007). Therefore, once a presumptive diagnosis has been made on the basis of signalment, clinical signs, and supporting clinicopathological features, supplementation should be started immediately, without waiting for the results of a $\mathrm{T}_{4}$ analysis (Atkinson and Aubert, 2004). The affected dog in this case had a good response to the therapy, resolving clinical signs, clinicopathological changes, and abnormalities on thyroid tests.

To the authors' knowledge, this is the first report describing the clinical and laboratory features and the successful treatment of hypothyroidism concurrent with myxedema coma in veterinary medicine of Korea.

\section{REFERENCES}

Atkinson K, Aubert I. 2004. Myxedema coma leading to respiratory depression in a dog. Can Vet J 45: 318-320.

Blois SL, Poma R, Stalker MJ, Allen DG. 2008. A case of primary hypothyroidism causing central nervous system atherosclerosis in a dog. Can Vet J 49: 789-792.

Chastain CB, McNeel SV, Graham CL, Pezzanite SC. 1983. Congenital hypothyroidism in a dog due to an iodide organification defect. Am J Vet Res 44: 1257-1265.

Dixon RM, Reid SW, Mooney CT. 1999. Epidemiological, clinical, haematological and biochemical characteristics of canine hypothyroidism. Vet Rec 145: 481-487.

Dixon RM, Reid SW, Mooney CT. 2002. Treatment and therapeutic monitoring of canine hypothyroidism. J Small Anim Pract 43: 334-340.

Ferguson DC. 2007. Testing for hypothyroidism in dogs. Vet Clin North Am Small Anim Pract 37: 647-669.
Finora K, Greco D. 2007. Hypothyroidism and myxedema coma. Compend Contin Educ Vet 29: 19-31.

Fliers E, Wiersinga WM. 2003. Myxedema coma. Rev Endor Metab Disord 4: 137-141.

Graham P. 2009. Canine hypothyroidism: diagnosis and therapy. In Practice 31: 77-82.

Graham PA, Refsal KR, Nachreiner RF. 2007. Etiopathologic findings of canine hypothyroidism. Vet Clin North Am Small Anim Pract 37: 617-631.

Henik RA, Dixon RM. 2000. Intravenous administration of levothyroxine for treatment of suspected myxedematous coma complicated by severe hypothermia in a dog. J Am Vet Med Assoc 216: 713-717.

Jaggy A, Oliver JE, Ferguson DC, Mahaffey EA, Glaus T Jr. 1994. Neurological manifestations of hypothyroidism: a retrospective study of 29 dogs. J Vet Intern Med 8: 328336.

Mesich ML, Mayhew PD, Paek M, Holt DE, Brown DC. 2009. Gall bladder mucoceles and their association with endocrinopathies in dogs: a retrospective case-control study. J Small Anim Pract 50: 630-635.

Mooney CT. 2011. Canine hypothyroidism: A review of aetiology and diagnosis. $\mathrm{N}$ Z Vet J 59: 105-114.

Panciera DL. 2001. Conditions associated with canine hypothyroidism. The Vet Clin North Am Small Anim Pract 31: 935-950.

Park S, Chatterjee V. 2005. Genetics of congenital hypothyroidism. J Med Genet 42: 379-389.

Pullen WH, Hess RS. 2006. Hypothyroid dogs treated with intravenous levothyroxine. Journal of Veterinary Internal Medicine 20: 32-37.

Scott-Moncrieff JC. 2007. Clinical signs and concurrent diseases of hypothyroidism in dogs and cats. Vet Clin North Am Small Anim Pract 37: 709-722.

Yamamoto T, Fukuyama J, Fujiyoshi A. 1999. Factors associated with mortality of myxedema coma: report of eight cases and literature survey. Thyroid 9: 1167-1174. 